\title{
How to check a computer program
}

\author{
SETH ROBERTS \\ Brown University, Providence, Rhode Island 02912
}

\begin{abstract}
The usual ways of checking computer programs have important flaws. A better way to check a program you have written is to have someone else write a program that does the same thing and then compare the output of the two programs over a wide range of input. Two examples are described.
\end{abstract}

By "checking" a computer program, I mean checking it for errors. The programs that psychologists write, such as programs for running experiments or analyzing data, are almost always checked (1) by hand or mental computation, or by comparison with a published example, and (2) by the same person who wrote them. The problem with the first feature is that not many test cases can be tried; the test input will probably vary much less than the actual input. The problem with the second feature is that the same errors can easily occur in both the program and the check. But both features are more or less standard practice. This may be one reason that programs believed to be completely correct sometimes contain serious errors (e.g., Gerhart \& Yelowitz, 1976; Myers, 1976, pp. 25.26).

A much better way to check a program you have written (better because it avoids these problems) is to have another person write a program that does the same thing as your program and then compare the output of the two programs over a wide range of input. It is easy to try a wide range of input because no hand or mental calculation is involved. Because the programmers are different, it is unlikely that the two programs will be wrong in the same way. When the two programs agree, the original program is probably correct. I describe here two examples of the use of this method.

The experiments of Roberts and Church (1978) were controlled by a computer. The procedure of the first experiment was complicated. To check the program for the procedure, we wrote the program twice. Church wrote the program in the language used for controlling experiments (TIICIE; Dyckman \& Church, 1972); I wrote the program in APL. We then entered the same random numbers to guide probabilistic decisions within the programs and simulated the responses of the subject according to the same rules. Mainly because the two programmers were different, but also because the two programming languages were different, we assumed that an error in the TIICIE program was unlikely to be matched by an error in the APL program. The two programs initially produced different output; it turned out that

I thank Russell Church for his help. This work was supported by NSF Grant BNS79-08839-01. Address reprint requests to Seth Roberts, who is now at the Department of Psychology, University of California, Berkeley, California 94720. both had mistakes. When the two programs finally agreed, we concluded that the TIICIE program was correct. This conclusion was supported by our experience using the program-We never found any mistakes.

We used the same method to check a program for data analysis. I had written a program in BASIC to find row-PLUS-column fits for two-way tables using a procedure described by McNeil and Tukey (1975). (Our main use of this procedure was to find averages better than the mean or median.) After 40 or 50 uses, it had become clear that the program was very useful, and it was worthwhile to check it carefully. So Church wrote another program (in BASIC) to do the same thing. The original program turned out to be correct, in the sense that no changes in it were needed for the two programs to agree. But the effort of writing the second program was not wasted. Three things were gained: (1) Our confidence in the original program increased considerably. (2) The program computed the fits by iteration, and in the course of finding the faults in the new program, we noticed an unexpected problem with the criterion used to stop iteration. (Our criterion was somewhat different from the criterion used by McNeil and Tukey, 1975.) This was not a flaw in the program but a flaw in the algorithm of which the program is a translation. (3) The implementation of BASIC we used (supplied by Digital Equipment Corporation for the RT-11 operating system, BASIC Version 1) prints numbers to six digits. When the programs appeared to agree, it was only to five digits. The programs used floating-point arithmetic, and presumably the differences in the sixth digit were due to differences in the order in which calculations were done. The fact that the agreement was to five digits gave us some idea of the error in the floating-point arithmetic; measurements of the error in a sequence of floatingpoint operations are in general hard to come by (Knuth, 1969, pp. 195-208). (After we did this, a doubleprecision version of BASIC came out. We could now judge the error by running the program with both single and double precision.) As in the first example, the conclusion that the original program was correct was supported by later events; after about 1,000 uses, no errors have been found.

Program checking is a subject with a large literature (e.g., Myers, 1976, 1979; Yeh, 1977), but there is a 
simple reason why this method of checking should be important. To check anything, you must essentially replace it. If there is a flaw in a certain part of a system, you can discover the flaw only if that part is replaced. To find a flaw in a program caused by the person who wrote the program, you must somehow replace that person. Ordinary methods of checking do this only in a very limited way: They replace the person who wrote the program with the same person in a different situation.

\section{REFERENCES}

Dyckman, H. L., \& Church, R. M. The TIICIE system for interactive control of independent experiments. In Computers in the psychology laboratory (Vol. 2). Maynard, Mass: Digital Equipment Corporation, 1972.

Gerhart, S., \& Yelowitz, L. Observations of fallability in applications of modern programming methodology. IEEE Transactions on Software Engineering, 1976, 2, 195-207.

KNUTH, D. E. The art of computer programming (Vol. 2). Reading, Mass: Addison-Wesley, 1969.

MCNE1L, D. R., \& TUKEY, J. W. Higher-order diagnosis of twoway tables, illustrated on two sets of demographic empirical distributions. Biometrics, 1975, 31, 487-510.

Myens, G. L. Software reliability: Principles and practices. New York: Wiley, 1976.

Myers, G. L. The art of software testing. New York: Wiley, 1979.

Roberts, S., \& Chunch, R. M. Control of an internal clock. Journal of Experimental Psychology: Animal Behavior Processes, $1978,4,318-337$.

YEH, R. T. (Ed.). Current trends in programming methodology (Vol. 2). Englewood Cliffs, N.J: Prentice-Hall, 1977. 